\title{
Examining the Role of Personality Factors in Problematic Video Game Play Associated with Facebook Games
}

\author{
Steve J. Groves, Jason L. Skues, Lisa Z. Wise \\ Department of Psychological Sciences, Swinburne University of Technology, Melbourne, Australia \\ Email: Sgroves@swin.edu.au
}

Received 22 May 2015; accepted 6 July 2015; published 9 July 2015

Copyright (C) 2015 by authors and Scientific Research Publishing Inc. This work is licensed under the Creative Commons Attribution International License (CC BY). http://creativecommons.org/licenses/by/4.0/

(c) $\underset{\mathrm{EY}}{\mathrm{B}}$ Open Access

\section{Abstract}

The current study used the structural components of Facebook games to select predictors of Problematic Video Game Play (PVGP) in relation to Facebook games. Based upon how game design and structure may interact with personality characteristics, the current study examined the effects of hypercompetitiveness, narcissism, reward responsiveness and psychological absorption on PVGP for a group of Facebook gamers. Moreover, the present study also observed how results may differ between Facebook gamers $(n=102)$, and a group of other (non-Facebook) gamers $(n=$ 132). The Facebook group comprised $84 \%$ of females with an average age of 40.39 (SD = 14.00), whereas the other group of gamers consisted of $61 \%$ of males with an average age of 24.70 (SD = 7.86). Participants completed an online survey. Structural Equation Modelling (SEM) revealed that an interaction between hypercompetitiveness and narcissism was the only significant predictor of PVGP for Facebook gamers. In contrast, a second structural model revealed that both hypercompetitiveness and psychological absorption were significant predictors of PVGP for other types of gamers. The findings from this study had important implications regarding the structure of video games and demographic differences between Facebook Gamers and other types of gamers.

\section{Keywords}

Video Games, Problematic Video Game Use, Social Networking Games, Facebook Games, Video Game Addiction

\section{Introduction}

A substantial body of research has emerged over the past decade that has focused on an individual's propensity

How to cite this paper: Groves, S.J., Skues, J.L. and Wise, L.Z. (2015) Examining the Role of Personality Factors in Problematic Video Game Play Associated with Facebook Games. Social Networking, 4, 80-95. 
to develop a behavioural dependence or "addiction" to video games [1]. Several terms have been used to describe this phenomenon; some of the more frequently occurring terms include "Internet gaming addiction" [2], "Video game addiction" [3] and Problematic Video Game Play [4]. The authors use the term Problematic Video Game Play (PVGP), to refer to such studies collectively, and define PVGP within the context of Griffiths' components approach [5] as a combination of pathological experiences with video games (e.g. loss of control over gaming and unpleasant feelings when is unable to play) and negative outcomes which result from conflicts between video game use and other aspects of one's life. Research focuses on the prevalence of PVGP and relates issues estimates that between $1.7 \%$ and $9.3 \%$ of video game players experience such problem game behaviours [6]-[10].

Whilst there have been several studies focused on individual differences which may lead some players to becoming more susceptible to developing PVGP than others (e.g. [11]-[13]), there appears an increasing body of literature focused on how video game content and design may also influence players. That is, although players may perceive that they are in control over their own motivations for playing, it is important to explore how video games may be designed in a way that encourages problematic engagement, regardless of the player's perceived motivations for playing. In response to this notion, King, Delfabbro and Griffiths [14] propose a psycho-structural taxonomy of structural characteristics common in video games which may encourage PVGP. King and colleagues organised these components into five broad categories, namely 1) social features, which relate to components that encourage player interaction and competition; 2) manipulation and control, which relate to the player's freedom to move and customise elements of the game; 3) reward and punishment features, which relate to reward schedules and mechanisms of performance feedback; 4) narrative and identity features, which focus on features such as story, and character development; and 5) presentation features such as graphics and sound.

In a recent study, Groves, Skues and Wise [15] use King et al.’s [14] taxonomy to assess how these structural components may influence player engagement in two popular genres of games played on Facebook, namely (1) Tile-Matching games, a type of game where the player must solve a series of puzzles by matching coloured blocks (e.g. Candy Crush Saga), and (2) Simulation RPGs, a type of game where players manage a small space by upgrading equipment and producing resources (e.g. Farmville). Ten of the most popular Facebook games fitting either genre are played by one of the authors, and features which have any potential impacts on engagement or PVGP are noted and explored qualitatively. Findings of this study suggest that both genres use reward schedules, such as limiting how long individuals can play and making players wait before they can return in order to engage players and encourage future investments in the game. It is argued that this may encourage PVGP as limiting when and for how long individuals play may lead to conflicts with one's other responsibilities (such as work or sleep), and that if players are motivated enough by in-game rewards, and they may sacrifice such responsibilities at the expense of playing the game. Moreover, Groves and colleagues also note several social aspects of Facebook games promoted a competitive atmosphere by emphasising and comparing one's in-game achievements with others. It is suggested that this promotes a competitive atmosphere that can potentially become problematic if such competition are taken too seriously.

Although an in depth qualitative study of Facebook games is useful in understanding how such games may influence individuals, as very few studies have explored PVGP within the context of Facebook games, Groves et al.'s [15] assertions have not yet been supported empirically. This is a significant gap in the literature given that more than 250 million people play Social Networking Games on Facebook every month [16], with some of the more popular Facebook games having more than 10 million daily active users [17]. Moreover, there is also very little known about the types of people who play Facebook games, or those who may be more susceptible to developing PVGP than others within this context. For example, if Facebook games do in fact employ certain game mechanics to encourage player engagement, then it is possible that differences in personality characteristics which are influenced by these mechanics will have an impact on PVGP behaviours.

Though several studies have highlighted the need to combine research on how game structure may influence different personality characteristics [15] [18] [19], very few studies have actually done this and none have attempted to do this in relation to Facebook games. To this end, the current study will use knowledge about the structure of Facebook games to inform the selection of personality traits and how these will be associated with PVGP for Facebook game use. The next section will discuss four personality factors that were based on understandings of the structure of Facebook games and are expected to predict PVGP within the individuals who play such games, namely hypercompetitiveness, narcissism, reward responsiveness and psychological absorption. 


\subsection{Hypercompetitiveness}

Groves et al. [15] highlights several aspects of Facebook games which encourage a competitive environment by using members of the player's own personal social network. For instance, Tile-matching games use a path-like representation of overall game success over time, which shows both what level the individual has reached, as well as how far ahead (or behind) their Facebook friends are by comparison. Similarly, Simulation/RPGs tend to display Facebook Friends' overall levels at the bottom of the screen at all times, again comparing long term progress. Groves et al. argued that this constant comparison to other players may encourage an individual to play more often to either catch up to their peers, or to remain on the top of leaderboards. This has significant implications in terms of one's ability to withdraw from the game, preoccupied thoughts about returning to the game, and relapse if that individual were in the midst of trying to cut down on playing time.

Assuming that competition does drive players to return more frequently or more often to Facebook games, it would be expected that individuals with a win-at-all costs attitude may place such value on being the best of their Facebook friends that they may be more inclined to sacrifice other aspects of their lives (e.g. sleep or work commitments) instead of reducing their play time or engagement with the game, even if this is at the expense of their own mental health (conflict). While there have been studies linking competitiveness motivations behind playing Facebook games [20], and more generally there appears an association between video game use and competitiveness [21], such conceptualisations do not necessarily capture competitiveness over and above general competition, nor do they address the relationship between such competitive natures and PVGP. For this reason, the current study investigated the relationship between hypercompetitiveness: a trait associated with the need by individuals to compete and win at any costs [22] [23], and PVGP.

\subsection{Narcissism}

Similar to hypercompetitiveness, social features of Facebook games which facilitate a competitive environment in which success can be easily measured and compared with scores may entice certain individuals who have a desire for admiration from their peers, being better than others, and a high regard for self-presentation. Indeed, a major element of Simulation/RPGs such as Farmville and Coasterville revolves around decorating one's property or personal game space and visiting other player's spaces. As expected, the more prestigious and decorative items are often the most difficult to obtain, and may require dedicated, goal-directed play for several weeks, even months to attain [15]. Likewise, higher scores and status within Tile-Matching games also relate to more long term goals, as success in these games are represented by overall number of levels completed. In both instances, if an individual had the propensity or the desire to appear more competent than others, they would need to play more often than others to reach their goals faster, and as a result may view any available time where they did not play as a failure to capitalise on available play time. As a result, attempts to capitalise on available gaming time may lead to the game becoming a disproportionately important factor in one's life (salience), and could also result in distress or frustration when the player is prevented from returning to the game (withdrawal). This could be especially problematic if one's self-worth were tied to their in-game success, as the player may come to use good items or high levels as a personal marker of self-competence.

Based on the assumption that Facebook games do in fact encourage players to place value within having better items or in-game status than others, then it is assumed that a personality trait such as narcissism which is related to grandiosity, desires for admiration and an inflated sense of self-entitlement [24], would be a significant predictor of PVGP within a Facebook gaming population. In support of this view, previous research within an MMORPG population suggests the admiration and social recognition players received from other players drive narcissistic players to both spend more time playing, and resort to any means to achieve this superior status [25]. Although this has not been explored in the context of Facebook games, Facebook games do provide an environment where a player's Facebook friends can see their in-game success, and narcissistic individuals may be driven to appear superior to other players to the extent that they employ a more problematic play style in order to gain an advantage over others.

Not only has it been argued that hypercompetitiveness and narcissism should individually predict PVGP in Facebook games, it is plausible that these two personality factors interact to influence game playing behaviour. For example, hypercompetitive and highly narcissistic players are highly motivated to achieve high scores/beat their friends at the game, and also place great importance and value on being perceived as better than others respectively. As a result, these players would place even greater emphasis on in-game success, and perhaps be 
even more motivated to achieve in-game success despite the external costs. Therefore, the current study will examine both the individual and combined effects of hypercompetitiveness and narcissism on PVGP within the context of Facebook games.

\subsection{Reward Responsiveness}

In addition to features which encourage competition, Groves et al. [15] emphasised how different reward mechanics common in Facebook games may influence the development and maintenance of PVGP. It was noted that not only did such games provide a long term, consistent source for reward, but they also imposed some limitations in that players could only play so often and would frequently have to wait before they could continue their session. This created situations where the player was close to achieving a milestone or goal and was subsequently motivated to complete the task, but had to wait several hours before they were permitted to return to the game.

With such focus on reward, it would be reasonable to suggest that reward responsiveness should be investigated within the context of PVGP in Facebook games. Reward responsiveness is a key conceptual component of Gray’s "Behavioural Approach System” (BAS) [26], which refers to the tendency to respond positively to the occurrence or anticipation of a reward [27]. That is, those with a greater responsiveness to reward tend to seek out or approach rewarding stimuli more frequently, and with more positive affect [28]. In the context of Facebook games, it could be suggested that features which make players wait to return to the game may have a greater behavioural impact on those who are more responsive to reward, as their desire to return to the game to obtain an object of value or complete a level may be far greater than less responsive individuals. This could result in players choosing to play the game over other important life commitments (conflict), a strong desire to return to the game when unable to (withdrawal), and the need to play more frequently to experience more frequent in-game rewards (tolerance). As such, it is argued that greater reward responsiveness would relate to higher occurrences of PVGP since individuals who actively seek rewarding stimuli may be more susceptible to game features which use reinforcement strategies to encourage future play sessions.

\subsection{Psychological Absorption}

This desire to "escape" or the motivation to pass time appears very frequently as a predictor of PVGP within the video game literature [2] [4], and time loss has been linked with self-reported negative outcomes for gamers [29], however few studies have explored propensities, or psychological tendencies which underlie how individuals "lose track" of time. As video games could be viewed as an immersive medium, one possible explanation is that some players become so engrossed in the game that they fail to attend to surrounding stimuli, such as the passage of time. That is, if an individual had a predisposition to immersive tendencies, they may be more prone to losing track of time, and may therefore play for much longer than both their awareness and intention, potentially resulting in real world consequences.

In this regard, the current study will investigate the role of psychological absorption, which can be defined as the tendency to focus exclusively on a single task to the point where one is phased out from their surroundings [30]. The relationship between psychological absorption and PVGP would expectedly be more evident in games such as Facebook games, which take much longer to complete and can be played for long periods of time due to their repetitive game design. While this long term, repetitive play style is not new or exclusive to Facebook games (many MMORPGs operate on similar principles; see Karlsen, 2011 [31] for a discussion on “grinding”), Facebook games differ in that they are much more portable as they can be played on mobile devices, and therefore do not require a dedicated space (such as a computer) to play. It is argued here that this may increase instances of unintended conflict with other areas of one's life more-so than other types of games, as player's may play such games just before they intend to sleep or while at work to pass a few minutes, but may find themselves playing for much longer, resulting in a loss of sleep/work conflicts.

\subsection{Aims and Hypotheses}

The primary aim of the current study was to address a significant gap in the literature and examine predictors of PVGP in relation to Facebook games. To do this, knowledge about structural components of Facebook games were used to inform the selection of personality variables that would be associated with PVGP in the context 
Facebook game use. In addition, a secondary aim was to compare the similarities and differences in predictors of PVGP for a group of Facebook gamers with other types of gamers. Although it was argued here that predictors informed by the structure Facebook games would likely relate to PVGP in those who play such games, it is necessary to explore how these same relationships across a non-genre-specific population, as the structural characteristics outlined to encourage PVGP in Facebook games may be common (or even universal) across all types of video games. For example, it is possible that competition is a universal element found across most types of video games, thus it would be expected that hypercompetitive individuals are more likely to develop PVGP, regardless of which type of game they choose to play. It is for this reason that the current study also explored how such findings would compare to other types of gamers. It was hypothesised that:

(H1) Hypercompetitiveness, narcissism, reward responsiveness and psychological absorption would each be positively associated with PVGP related to the use of Facebook games.

(H2) Hypercompetitiveness and narcissism would interact to influence PVGP related to the use of Facebook Games.

(Exploratory) Compare and contrast personality predictors of PVGP for individuals who play Facebook games with a separate group of individuals who play other (non-Facebook) types of video games.

\section{Method}

\subsection{Participants}

Participants were primarily recruited through online forums and Facebook. To ensure that both those who played Facebook games and those who played other types of games were evenly represented in the sample, a link to the online survey was posted on several Facebook game fan-pages and groups (e.g. Candy Crush Saga fan pages), as well as more general video game-related forums (e.g. Reddit pages such as r/gaming). A secondary purpose of recruiting in such places was to ensure the sample included dedicated players, which the authors argue here is important for studies exploring more problematic use as the game is important enough to their life that they actively involve themselves in communities. Snowball sampling [32] was encouraged amongst these participants and communities. Undergraduate university students were also recruited through a research experience program, where first year undergraduate psychology students receive coursework credit for signing up to participate in studies.

The sample consisted of 246 participants who had played either Facebook games or any type of other video games for at least one hour, or at least five times in the past week. As the study was concerned primarily with individuals who experience PVGP, the above criteria were used to ensure only regular and frequent players were included in the sample. Moreover, it would be difficult to imply one's personality had any influence over an activity they do very rarely, and individuals who play video games less than the criteria may confound findings (e.g. an individual who is very competitive is unlikely to develop PVGP if they only play video games very occasionally). In terms of demographics, 57.6\% of participants were female, and ages ranged from 18 to 65 years with a mean age of $31(S D=13.34)$. Most of the sample resided in Australia $(62 \%)$, and $20 \%$ of the sample were from United States. The remaining 18\% of the sample were located across 20 different countries (e.g. United Kingdom, New Zealand, Philippines), with no country representing more than $2 \%$ of the total sample. Approximately $34 \%$ of the participants in the sample were employed full-time, with 16\% employed part-time and $19 \%$ employed on a casual basis. In terms of education status, $39 \%$ of the sample were current undergraduate students, 11\% were current postgraduate students, 6\% were currently attending TAFE (Technical and Further Education) or similar tertiary courses (i.e. Vocational, trade schooling). Thirty eight percent were not current students.

\subsection{Materials}

Participants completed a 15 - 20 minute online survey which measured hypercompetitiveness, narcissism, reward responsiveness, psychological absorption and PVGP. Demographic questions and questions about the frequency and duration of time spent playing Facebook games including different types of Facebook games as well as other game genres (e.g. MMORPGs and console games) were also asked. Each of the psychometric measures used are presented below.

\subsubsection{Problematic Video Game Play Test [33]}

PVGP was measured in this study using the Problematic Video Game Play Test (PVGT) [33]. The PVGT is a 20 
item self-report measure which consists of questions relating to the frequency of problem behaviours or outcomes associated with problematic use (“do you lose sleep due to late night video game playing?”), with responses ranging from 1 = "Never" to 5 = "Always". Higher scores indicate greater amounts of PVGP behaviour. The initial validation of this scale demonstrated good internal consistency, as observed by a high coefficient alpha [34] ( $\alpha=0.92)$, a commonly used test of reliability within social sciences $(\alpha>0.7$ is often considered adequate fit).Moreover, reviews of available psychometric tools show this measure to align well with both definitions/conceptualisations of PVGP and other existing measures [35] [36].

\subsubsection{Hypercompetitive Attitudes Scale [23]}

Hypercompetitiveness was measured using the Hypercompetitive Attitudes Scale (HCA) [23]. The HCA is a 26 item self-report scale in which respondents must answer questions about their competitive attitudes (e.g., "I compete with others, even if they are not competing with me"). Responses range from 1 = "Never true of me" and 5 = "Always true of me". Approximately half of the items were reverse coded, and higher scores indicate greater levels of trait hypercompetitiveness. It should be highlighted that participants were also prompted before the HCA to answer the questions thinking specifically about their interactions with others in video games, and the term "athletic competition" was changed to simple "competition" in two of the items, to ensure questions were context of the current study. The HCA reported good internal consistency ( $\alpha=0.91)$ within the validation study, and other studies using this measure have also displayed good internal consistencies [37] [38].

\subsubsection{Narcissistic Personality Inventory, 16 Item Version [24]}

Trait narcissism was measured using the Narcissistic Personality Inventory, 16 item version (NPI-16) [24], which is a shortened version of the 40-item Narcissistic Personality Inventory (NPI40) [39]. Participants were presented with two statements: one narcissistic and another non-narcissistic (e.g. "I am an extraordinary person" vs. "I am much like everybody else" respectively), and must select which of the two statements they think best describes them. 1 point was allocated to the endorsement of a narcissistic statement, and 0 points for endorsement of the non-narcissistic statement. Higher scores represent greater narcissism. The initial validation of the NPI-16 displayed adequate internal consistency $(\alpha=0.72)$ as well as good discriminant and predictive validity, with narcissism scores correlating with individual's tendencies to rate themselves as more powerful, influential and attractive than others.

\subsubsection{Attentional Resource Allocation Scale [30]}

Psychological Absorption was measured using items from the Attentional Resource Allocation Scale (ARAS) [30]. The ARAS is a 15-item self-report composite measure for trait absorption and dissociation. Participants are asked to respond to a series questions and statements about experiences or moments related to attention, or lack of attention to present stimuli ("When I listen to music I can get so caught up in it that I don't notice anything else") with responses measured on a 5-point scale ranging from $1=$ "never" to $5=$ "always". The ARAS contains 3 sub-factors, but only the absorption factor (6 items) was used in the current study. The initial validation study revealed adequate internal consistency $(\alpha=0.74)$ for the absorption factor.

\subsubsection{BIS/BAS Scales}

Reward Responsiveness was measured using items from Carver and White's BIS/BAS scales [27]. The BIS/BAS scales are a collection of 24 items used to measure different facets of Gray's (1987) [26] theoretical "Behavioural Approach System" (BAS) and "Behavioural Inhibition System" (BIS). Participants are asked to respond using a 4-point scale ranging from 1 = very true of me, to 4 = very false of me. As all items except 2 and 22 (which are negatively worded) are reverse scored, and higher scores indicate greater BIS or BAS. The reward responsiveness factor (5 items) was the only factor used in the current study. The validation study suggested this factor displays good internal consistency, and similar results have been replicated in more recent studies [40] [41].

\subsubsection{Frequency and Duration of Video Game Use}

Frequency and duration of game use were measured by a series of matrices regarding player behaviours from the prior week. Participants were asked to self-report both how often they had started playing a video game, as well as how long they had spent playing that day for 6 different types of video games for each day within the previous week. Answers for each day of the week were summed to give a weekly score for both frequency of play, 
and for time spent playing. Of these 6 game types, three were Facebook genres (Tile-Matching, Simulation/RPG and other), and three were types of common non-Facebook gaming experiences (MMORPGs, other MMO’s and other games).

\subsection{Procedure}

Participants were asked to complete an online questionnaire, which was no longer than 30 minutes. This questionnaire included all measures listed above, as well as questions about demographics and video game use. Although students received course credit for agreeing to participate, this course credit was only minor (e.g. one extra point added to the final grade), and students were credited for merely signing up, even if they did not complete the questionnaire. The authors agreed this incentive was not substantial enough to require an equivalent incentive for non-student participants.

\subsection{Statistical Design}

Structural Equation Modelling (SEM) was used to analyse the data. Firstly, one-factor Confirmatory Factor Analysis (CFA) models were used to address any measurement issues associated with each of the variables of interest. Items that did not fit the data were removed and not included in the composite scores or structural model. Secondly, a structural model was tested in which hypercompetitiveness, narcissism, reward responsiveness and psychological absorption were the independent variables, and PVGP was the dependent variable. Only independent variables mentioned that correlated with PVGP were included in the model. In addition, in order to test interactions, the current study used the Unconstrained Latent Variable Approach [42] to test interactions in SEM, where each of the independent variables was centred and a product term was created to represent the interaction. Consistent previous research [42] two indicators were used for each of the latent interactions.

Models were tested for both a Facebook gaming population, and a group of individuals who played other, non-Facebook games, so that differences in the models could be compared. Given that all variables were of a unidimentional structure, and taking account that the 246 participants would be split in two groups yielding relatively small sample sizes, single indicator latent variables (SILVs) [43] were used to operationalise these constructs within the models. Not only does this approach allow measurement error to be accounted for, it also reduces the number of parameters to be estimated in the model and therefore is an appropriate strategy for dealing with small sizes.

\section{Results}

Prior to analysis, data were screened for any missing or out-of-range values. After cases with more than $25 \%$ missing data were deleted listwise, a Missing Values Analysis (MVA) was performed using Little’s MCAR test, which confirmed that remaining missing data were missing completely at random $\left(\chi^{2}(7257)=7443.70, p=.062\right)$, and all remaining missing data were replaced using the Estimation Maximisation (EM) method. In terms of out-of-range values, 1 participant had reported overall playing video games for 10,050 minutes within the past week (23.97 hours per day). This was considered to be unlikely and the case was removed from further analyses.

\subsection{Measurement Models}

One factor CFA models were performed for each measure to determine whether the items associated with each of the variables provided an adequate fit to the data. Prior to analysis of the CFA measurement models, an initial Exploratory Factor Analysis (EFA) revealed two distinct factors found within the HCA: one containing all negatively worded items (13) and the other containing positively worded. It was decided that only the positively-worded items would be used in the current study, as the authors argue these items appeared to have more conceptual relevance to the construct of interest.

The CFA for reward responsiveness revealed an adequate fit to the data $\left(\chi^{2}(5)=11.16, p=0.05\right.$; CFI $=0.97$, SRMR = 0.04, RSMEA = 0.01). However, the CFAs for PVGP $\left(\chi^{2}(170)=670.8, p<0.001\right.$; CFI = 0.77, SRMR $=0.08$, RSMEA $=0.11)$, hypercompetitiveness $\left(\chi^{2}(77)=297.5, p<0.001\right.$; CFI $=0.0 .83$, SRMR $=0.07$, RSMEA $=0.11$.$) , narcissism \left(\chi^{2}(104)=373.67, p<0.001 ;\right.$ CFI $=0.66$, SRMR $=0.08$, RSMEA $\left.=0.10\right)$ and psychological absorption $\left(\chi^{2}(9)=30.89, p<0.001\right.$; CFI $=0.95$, SRMR $=0.05$, RSMEA $\left.=0.10\right)$ did not meet requirements for adequate fit. In this regard, model trimming was used in which items were removed systemati- 
cally from each of the scales according to an inspection of the size of the standardised residuals and the greatest impacts on the $\chi^{2}$ value associated with modification indices, as well as whether there was a plausible conceptual reason for item misfit until adequate fit was achieved. The final CFA models were PVGP $\left(\chi^{2}(65)=123.87, p<\right.$ 0.001; CFI = 0.94, SRMR = 0.05, RMSEA = 0.06); hypercompetitiveness $\left(\chi^{2}(35)=76.57, p<0.001\right.$; CFI = 0.94, SRMR $=0.05$, RMSEA $=0.07)$, narcissism $\left(\chi^{2}(14)=21.71, p=0.08\right)$, and psychological absorption $\left(\chi^{2}(9)=\right.$ 30.89, $p<0.001$; GFI $=0.95$, SRMR $=0.04$, RMSEA $=0.10$ ). The coefficient alphas for each of the measures are presented later in Table 1.

\subsection{Assumption Testing}

Univariate normality was tested using visual inspection of histograms and normality plots with standardised residuals and revealed that PVGP, hypercomptitiveness and psychological absorption were all normally distributed. In contrast, there was a mild negative skewness for reward responsiveness, and moderate positive skewness for narcissism. In addition, as both frequency and duration of video game use were both count variables, expectedly, both were strongly negatively skewed. These variables were left untransformed, and equivalent non-parametric tests were used where appropriate. In addition, a Bollen-Stine Bootstrap with 2000 iterations was used since this is a non-parametric resampling method which helps to address issues of non-normal data [43].

\subsection{Descriptive Statistics}

Data were obtained for each player's use of three categories of Facebook games (Tile-matching, Sim/RPG, and “other”), and three categories of non-Facebook games (MMORPGs, other MMOs, and other games). Since sample sizes were not large enough, and due to many participants playing multiple types of games, the sample was divided into two superordinate categories, namely (1) Facebook gamers who play any type of Facebook Games, and (2) Other gamers, whom played video games from any of the non-Facebook game categories. A small proportion of participants who played both Facebook and non-Facebook games $(n=45)$ were allocated to respective groups only if they spent twice as long playing one type of game than the other. Eleven participants played both Facebook games and other types of games equally as often, and were removed from further analysis.

In this study, time spent playing video games varied from 8 minutes to 5040 minutes (84 hours) per week. For Facebook gamers, the median time spent playing video games per week was 445 minutes or 7.4 hours $(M=$ 803.77, $S D=847.43)$, whereas other types of gamers played a median of 540 minutes or 9 hours $(M=768.64$, $S D=799.29)$. However, this difference was not significant $(p=0.995)$ according to a Mann-Whitney test. Although there were no significant differences in the overall time both groups spent playing video games, Facebook gamers reported playing more frequently $(M=43.78$ per, $S D=51.70$, Median $=26)$ than did other gamers $(M=14.74$ per, $S D=16.25$, Median $=10)$, and a Mann-Whitney test confirmed this difference to be significant ( $p<0.001)$. Table 1 displays the differences between group means on the independent and dependent variables, as well as the intercorrelations amongst the variables for both Facebook gamers and other gamers.

As can be seen in Table 1, there were very small differences in means between the two groups in terms of the

Table 1. Coefficient alphas, means, standard deviations, and correlates amongst the variables.

\begin{tabular}{|c|c|c|c|c|c|c|c|c|c|c|c|}
\hline \multirow{2}{*}{ Variables } & \multicolumn{3}{|c|}{$\begin{array}{c}\text { Facebook } \\
\text { Gamers }(n=102)\end{array}$} & \multicolumn{2}{|c|}{$\begin{array}{l}\text { Other Gamers } \\
(\mathrm{n}=132)\end{array}$} & \multicolumn{6}{|c|}{ Correlations } \\
\hline & $A$ & $M$ & $S D$ & $M$ & $S D$ & $\mathrm{TR}$ & 1. & 2. & 3. & 4. & 5. \\
\hline 1. PVGP & 0.88 & 2.27 & 0.74 & 2.32 & 0.66 & $1-5$ & -- & $0.315^{* * *}$ & $0.308^{* * *}$ & -0.027 & 0.050 \\
\hline 2.Hypercompetitiveness & 0.86 & 2.41 & 0.83 & 2.60 & 0.69 & $1-5$ & $0.409^{* * *}$ & -- & $0.380^{* * *}$ & $0.344^{* * *}$ & $0.393^{* * *}$ \\
\hline 3. Absorption & 0.76 & 2.33 & 0.88 & 2.43 & 0.82 & $1-5$ & $0.311^{* *}$ & $0.516^{* * *}$ & -- & 0.155 & $0.250^{* *}$ \\
\hline 4. Narcissism & 0.72 & 0.22 & 0.27 & 0.29 & 0.26 & $0-1$ & $0.201^{*}$ & $0.463^{* * *}$ & $0.357^{* * *}$ & -- & $0.241^{* *}$ \\
\hline 5.Reward responsiveness & 0.72 & 3.32 & 0.50 & 3.35 & 0.42 & $1-4$ & -0.024 & 0.102 & 0.056 & -0.032 & -- \\
\hline
\end{tabular}

$N=234, \stackrel{*}{p}<0.05, \stackrel{* *}{p} p<0.01,{ }^{* * *} p<0.001$. Note: Correlations for Facebook gamers are presented below the diagonal, and correlations for other gamers are presented above. Where one or more measures violate assumptions of normality, Spearman's Rho is used. 
independent variables and the dependent variable, and follow-up t-tests revealed these differences were not significant. An inspection of the correlations reveals that, for Facebook gamers, hypercompetitiveness, narcissism and psychological absorption were all positively associated with PVGP at a weak to moderate level. For the other gamer group, however, only hypercompetitiveness and psychological absorption were significantly associated with PVGP. Reward responsiveness did not correlate with PVGP for either group.

In addition to the correlations presented above, spearman's rho also revealed that, for other gamers, both time spent playing $(r s(132)=0.436, p<0.001)$ and frequency of play $(r s(130)=0.347, p<0.001)$ were positively related to PVGP, but only time spent playing correlated with PVGP $(r s(101)=0.319, p<0.01)$ for the Facebook gamers0. Moreover, there were also noteworthy demographic differences between the Facebook gamer group and their counterparts. The other (non-Facebook) gamer group reported a mean age of $24.70(S D=7.86)$, whereas in contrast, the mean age for Facebook gamers was $40.39(S D=14.00)$. An independent samples t-test confirmed this difference to be significant $(t(232)=10.15, p<0.001)$. In addition, whereas only $39 \%$ of other/non-Facebook gamers were female, $84 \%$ of Facebook gamers were female. Pearson's Chi-Squared confirmed this difference to be significant $\left(\chi^{2}(4)=60.79, p<0.001\right)$.

\subsection{Structural Models}

Using data from the 102 Facebook gamers, a structural model was specified to test whether hypercompetitiveness, narcissism or psychological absorption predicted PVGP. Reward responsiveness did not correlate with PVGP and was not specified in the model. The revised model provided an adequate fit for the data $\left(\chi^{2}(3)=15.15\right.$, Bollen-Stine $p=0.08$, and explained $41 \%$ of the variance in PVGP. Figure 1 displays the final model and parameter estimates.

As can be seen in Figure 1, only the interaction between hypercompetitiveness and narcissism positively predicted PVGP. Notably, whilst hypercompetitiveness did not reveal a significant direct effect, this relationship was approaching significance $(p=0.06)$ and is most likely a reflection of the small sample size. Both narcissism and psychological absorption did not predict PVGP. A plot of the interaction (Figure 2) suggests that at a combination of high hypercompetitiveness and high narcissism produced the greatest PVGP scores. However, at high levels of hypercompetitiveness, narcissism appears to have only a minor influence over PVGP scores.

To further explore potential differences between Facebook Gamers and a heterogeneous sample of other gamers, a second structural model using the remaining data obtained from other types of gamers (non-Facebook gamers) was specified. Given that only psychological absorption and hypercompetitiveness were correlated with PVGP for the sample of other gamers, reward responsiveness and narcissism were not included in the model. An estimation of the model revealed a negative error variance attached to one of the indicators of the hypercompetitiveness/narcissism latent interaction. While there are potentially appropriate solutions for such occurrences [44] [45], given that there were no specific predictions for an interaction for other types of gamers, the interaction

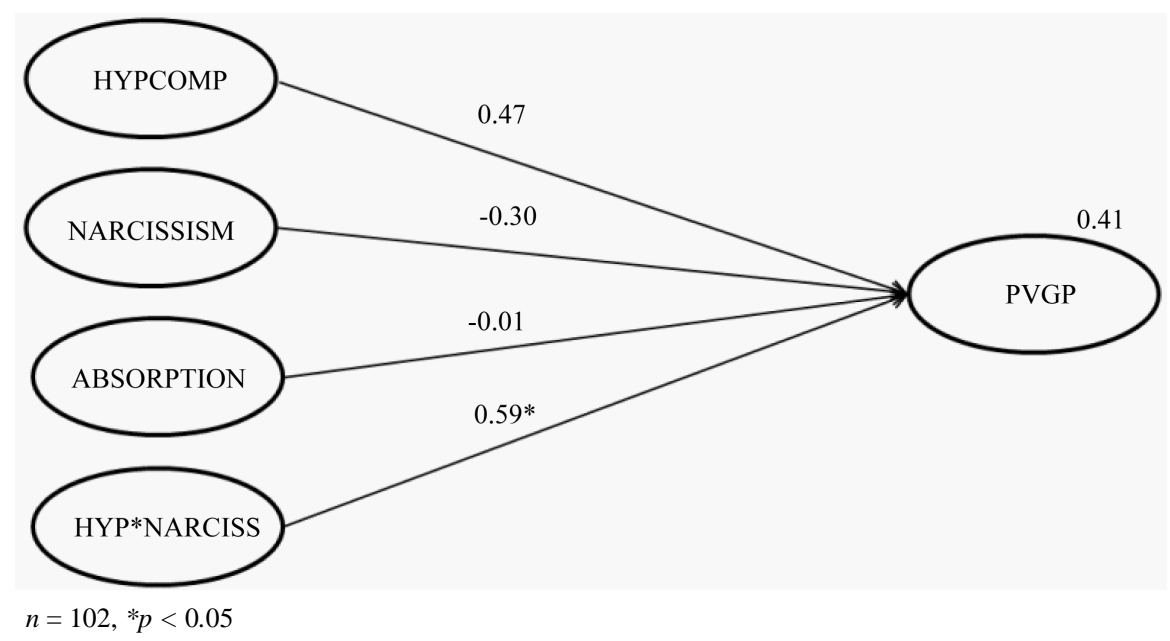

Figure 1. A structural model of hypercompetitiveness, narcissism, absorption, and problematic video game play in a sample of Facebook Gamers. 


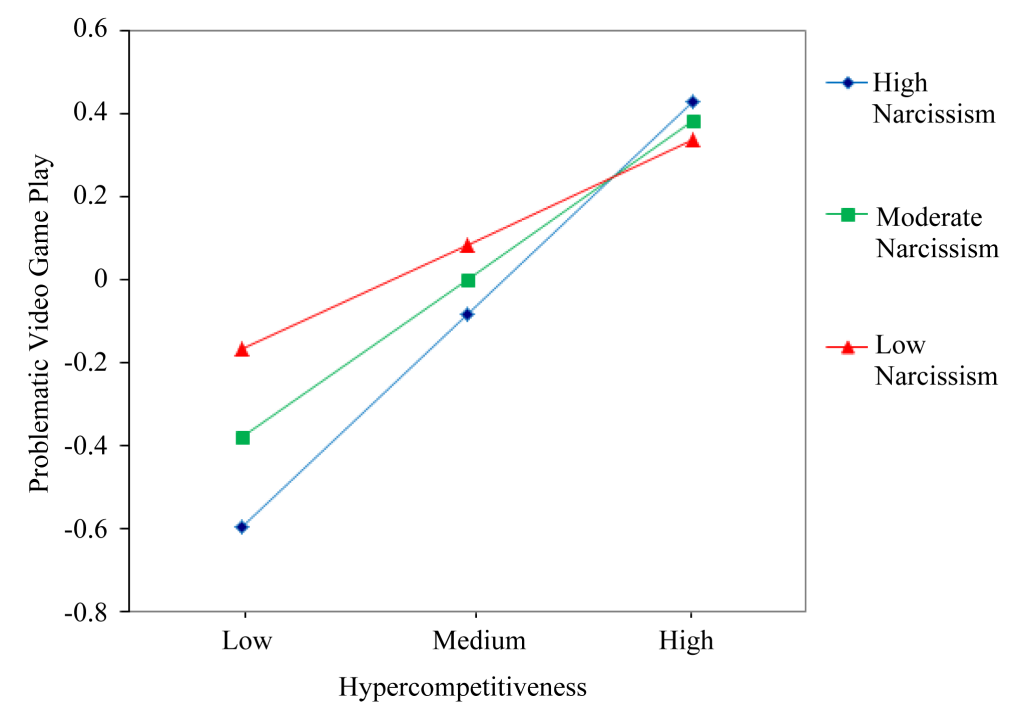

Figure 2. The interaction between narcissism and hypercompetitiveness on problematic video game play.

term was removed. A simple model with hypercompetitiveness and psychological absorption predicting PVGP was tested and explained 19\% of the variance in PVGP. No model fit indices were produced for the identified model. The model is displayed in Figure 3.

\section{Discussion}

\subsection{Interpretation of Results}

\subsubsection{Hypercompetitiveness and Narcissism}

Whilst both hypercompetitiveness and narcissism were significantly correlated with PVGP for Facebook gamers, there was no evidence that either predicted PVGP independently within this group when placed in a structural model with other variables. These findings are in contrast to the current study's hypothesis, and previous research, which suggests the design of Facebook games may influence PVGP through developing unhealthy competition [15], as well as previous research on narcissism within an MMORPG population [25]. Despite neither hypercompetitiveness nor narcissism significantly predicting PVGP in a sample of Facebook gamers, the interaction between these two did provide strong statistical evidence of their involvement with PVGP, thus supporting the hypothesis that narcissism and hypercompetitiveness would interact to predict PVGP. Somewhat consistent with the near significant findings pertaining to hypercompetitiveness and PVGP, an interpretation of the data suggests PVGP did increase alongside hypercompetitiveness. Further to this and consistent with predictions, the highest instances of PVGP occurred when both narcissism and hypercompetitiveness were also high. This would support the notion that PVGP within the context of Facebook games may be at least partially driven by design elements which encourage players to compare their achievements and compete with Facebook friends. However, as there was very little difference between PVGP scores across varying levels of narcissism when hypercompetitiveness was high, this interaction should be interpreted with caution.

Although hypercompetitiveness was not found to be a significant predictor of PVGP in the sample of Facebook gamers, it was significant in the structural model for the sample of other gamers. The authors make two suggestions as to why this may have occurred. Firstly, it is very likely that most types of video games facilitate at least some form of competition amongst players, and that this design aspect is common across many types of games. For instance, arcade games encourage highly competitive individuals to strive for high scores so that their names are displayed to others. Similarly, online computer games such as MMORPGs also display high scores and player skill levels to other players in the area. In fact, many video gaming consoles award players overall points for performing achievements or goals within each of their games [14], and these scores are totalled and displayed allowing players to compare their "gamerscores" (for Xbox) or "trophies" (for Playstation).

As for hypercompetitiveness and PVGP within the context of Facebook games only, the authors argue the 


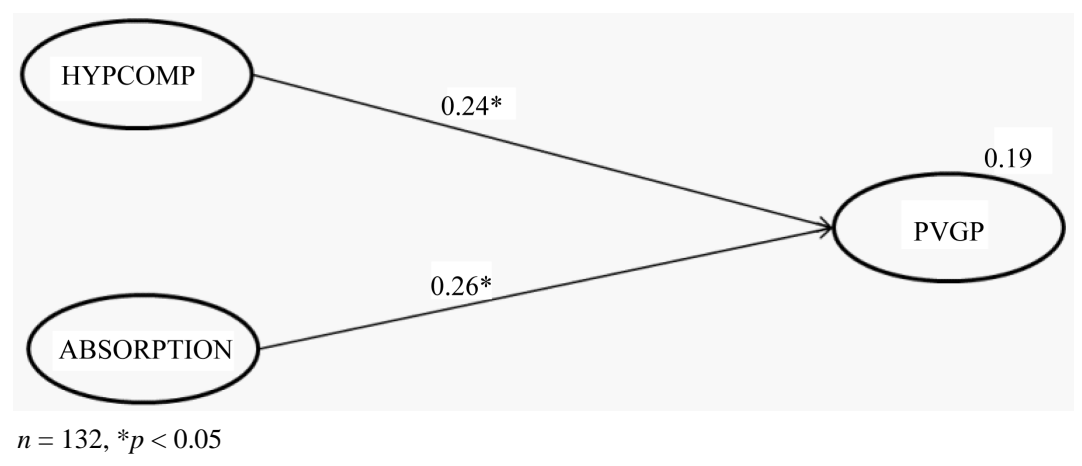

Figure 3. A structural model for hypercompetitiveness, absorption and problematic video game play in a sample of non-specific gamers (non-Facebook gamers).

lack of a significant finding may be an artefact of age and gender. Given that the average Facebook gamer was 40 years of age and female, it may be that people who play Facebook games may be reluctant to admit to playing video games as they do not fit the "gamer" stereotype [46]. This could lead Facebook gamers to having fewer peers to compete with, leading to less praise and social standing resulting from in-game success, and as a result players may be less driven to engage with Facebook games to a degree which becomes problematic, at least in terms of competitive reasons for playing. In addition, the label "gamer" may also be less salient to these individuals' identities, which may also lead players to boast less about their in-game achievements. Competitive individuals may seek environments where their achievements are praised and revered by others, thus Facebook gamers may look for other activities where their competitiveness leads to acknowledgement. However, as the relationship between hypercompetitiveness and PVGP was approaching statistical significance in the structural model for Facebook gamers, these findings merit further investigation.

In terms of narcissism and PVGP, this proposed link may relate to features not present in all types of Facebook games. Previous research [25] found narcissism to predict PVGP in an MMORPG sample, and it was suggested that this related to players using their avatar's appearance and social-standing as a means to denote higher status. Past research exploring addictive behaviours in reference to the Sim/RPG Facebook game Happy Farm [47] contended that players who were more materialistic would be more likely to develop addictive tendencies as a result of trying to gain extravagant or highly valued decorations or items. Likewise, narcissistic individuals playing Sim/RPGs may develop PVGP as a result of their desire for such items which denote superiority or social standing. Although Sim/RPGs Facebook games do have avatars and elements of customisation which can be used for presentation purposes, a majority of Facebook gamers observed in the current study mostly played TileMatching games: a genre which does not often include avatars. Though it was initially argued in the current study that this desire for superiority may extend to other aspects of in-game success (i.e. top positions on leaderboards), findings of the current study suggest this is not the case, and that players must also have some predisposition to compete with others for such features to promote PVGP.

\subsubsection{Psychological Absorption}

Although psychological absorption did not significantly predict PVGP for Facebook gamers as hypothesised by the current study, this relationship was significant for the other group of gamers. This is likely to be due to a function of design. For instance, as mentioned previously, Facebook games tended to only allow players to play in short bursts before they ran out of lives or resources and had to wait before they could play again. This feature may actually prevent PVGP in a Facebook sample, as players with a tendency to get absorbed in tasks may not notice how long they have spent playing, but running out of lives/resources prevents them from playing much longer than they had originally intended. This "short-bursts" style of gameplay offered by Facebook games may also explain the demographic differences observed within the current study, as differences in lifestyle factors (I.e. work, family responsibilities) may lead some individuals to prefer shorter, but more frequent sessions as opposed to playing a video game over a large block of time. The differences in lifestyle demands between these groups would need to be explored further before any conclusions can be made.

In addition, as Facebook games are often quite simplistic in both gameplay and depth [14], such games may not appeal to those with a proneness to being absorbed or immersed in tasks when compared with more in-depth 
and extensive games such as MMORPGs where gamers have an expansive world to explore. Video games where players must play in shorter bursts may not appeal as much to these individuals as they may find it more difficult to "lose themselves" within such a game. In contrast, players may find more in-depth games more immersive, and may choose to spend their free time absorbed in other games which facilitate this experience. For example, although a qualitative study exploring player experience found that players saw time loss as a negative outcome of video game use, the very same outcome was also viewed as a positive outcome by players (i.e. an activity which allows escape through time loss) [29]. This could be problematic as it may encourage an attachment to the game, or an association with the game as being one's source for escape. Escapism motivations have been linked to PVGP across several studies [2] [48], and highly immersive environments may provide the perfect escape for individuals with a propensity to become absorbed in tasks.

\subsubsection{Reward Responsiveness}

Contrary to expectations, reward responsiveness had no significant impact on PVGP for either Facebook gamers or the other gamer group. In order to explain this finding, it is important to highlight the difference between traits which may influence responsiveness to reward, and the underlying reasons for playing the game. That is, while players may be motivated by in-game rewards, their reasons behind such motivations may not be based around the actual instance associated with receiving the reward, but instead, the value of an in-game reward may lie behind the socially and personally constructed meaning behind this game reward [49]. For example, individuals may set themselves a goal to reach a certain level, or to obtain an item that can only be obtained through many hours of repetitive play [31]. In this regard, it is not necessarily the feelings associated with reaching the goal which keeps the player motivated, but instead it may be the adoration from their peers, the fact that reaching this goal may allow the player access to new game features, or even the esteem they associate with that goal.

\subsection{Implications}

The findings from the current study have significant theoretical implications for researchers, as well as practical implications for players and psychologists. In terms of theoretical implications, the findings from the current study suggest that Facebook gamers may be an entirely different population compared to people who have previously been considered to be "gamers" in the literature. In particular, Facebook gamers tended to be older than other gamers, and were predominately female. This has important implications for researchers in terms of who plays video games, and how literature draws meaningful comparisons across studies that focus on different types of gamers.

The current study also has implications for researchers in terms of forming predictions about player susceptibilities to the development and maintenance of PVGP. It appears that determining predictors based on the structural characteristics of the game may in fact be a useful way to explore predictors of PVGP which may not have been previously examined. As such, it is recommended that researchers analyse how video games operate and encourage players to engage in PVGP. In addition, future research may wish to explore PVGP within other video game genres that, like Facebook games, have also been largely overlooked within literature using a similar structurally-derived approach to selecting predictors. More in-depth analysis of all video games may provide a better understanding of PVGP, not only within the context of those specific genres, but patterns which appear regardless of genre.

In terms of practical implications, the findings of the current study suggest that player personality may be influenced by game structure. Therefore it is important that players are made aware of how games may be exploiting such traits to promote further gaming engagement, and that players think critically about how aspects of their personalities may facilitate problematic use. Moreover, clinicians should also work with clients to explore and discuss aspects of the game in order to identify specific structural characteristics which may be at the core of the client's PVGP. Not only would identifying how the game and player interact be valuable for both parties in understanding the issue, it may also help guide goal setting and possible interventions. As Facebook games appear to reach an audience not typically well-represented within gaming populations, this is particularly important as such individuals may also be less familiar with common elements of game design.

\subsection{Limitations}

First and foremost, the demographic disparities between Facebook gamers and other types of gamers need to be 
addressed. As specific Facebook Game fan pages and sites were targeted in the study's recruitment stage, the sample was not a randomised representation of the wider population. While this could be viewed as an issue in terms of generalisability, it is important to note that the purpose of this study was not to capture a complete understanding of all individuals who use Facebook games, but merely to explore factors which may contribute to PVGP. Thus, sampling in these locations, rather than across the general population was necessary to ensure that highly engaged/involved Facebook gamers were included in the study's sample, as this would likely increase the prevalence of PVGP. In other words, the authors recruited in online spaces were different types of gamers are generally found, and the results most likely represent actual demographic differences between Facebook gamers and other types of gamers, rather than there being a higher-order reason as to why such demographics are drawn to these gaming-related spaces. Nevertheless, future research should focus on exploring how age and gender may differ or influence PVGP across different types of gamers in order to determine whether such differences are driven by demographic differences in gaming populations, or whether these are a result of game design.

A second limitation of the current study is the lack of self-report measures related to structural characteristics. Although the current study used previous research on structure to develop hypotheses about individual difference variables and PVGP, participants were not explicitly asked about structural elements of games which impacted on their decisions to play. While this can be inferred by exploring differences in genre, not only would a very large sample be required to observe detailed distinctions between different structural components of genres, but players may likely play more than one type of video game. The current study did remove those who played both Facebook games and other types of games regularly from any analyses to avoid this, but this was only possible as there appeared very little overlap between these groups, and may not be possible in studies comparing types of games with an overlapping audience.

Future research should explore more directly how structure and personality may interact to lead to PVGP by operationalising structural components of games and personality variables within the same study so that any potential interactions between these can be directly explored. For instance, previous studies have developed a structural characteristics survey which measures the enjoyment, perceived importance, and the behavioural impact of a number of features common in video games [19]. Perhaps researchers should consider using or developing similar measures alongside personality measures in order to create statistical models which best capture how both players, and features of the game may facilitate the development and maintenance of PVGP.

\subsection{Conclusion}

In conclusion, the current study has made several important contributions to the research. Firstly, this study is the first to examine PVGP within a Facebook games context. Secondly, this study employs a new approach towards investigating PVGP by using the structural characteristics of Facebook games to inform the selection of predictors of PVGP. The current study find that differences in game structure do in-fact lead to differences amongst the predictors of PVGP. In particular, whilst the interaction between hypercompetitiveness and narcissism is the only significant predictor for PVPG for Facebook games, hypercompetitiveness and psychological absorption predict PVGP in a sample of other gamers. In addition, the current study also suggests that Facebook gamers may represent a different population of gamers which has not yet been explored within the context of PVGP literature. Future research should measure structural characteristics and personality traits and test these simultaneously within the same model, and also explore the impact of age and gender in order to gain a more in-depth understanding of how these variables interact to influence PVGP.

\section{References}

[1] Griffiths, M.D., Kuss, D.J. and King, D.L. (2012) Video Game Addition: Past, Present and Future. Current Psychiatry Reviews, 8, 308-318. http://dx.doi.org/10.2174/157340012803520414

[2] Kuss, D.J., Louws, J. and Wiers, R.W. (2012) Online Gaming Addiction? Motives Predict Addictive Play Behavior in Massively Multiplayer Online Role-Playing Games. Cyberpsychology, Behavior and Social Networking, 15, 480-485. http://dx.doi.org/10.1089/cyber.2012.0034

[3] Van Rooij, A.J., Schoenmakers, T.M., van den Eijnden, R.J., Vermulst, A.A. and van de Mheen, D. (2012) Video Game Addiction Test: Validity, and Psychometric Characteristics. Cyberpsychology, Behaviour and Social Networking, 15, 507-511.

[4] King, D.L. and Delfabbro, P.H. (2009) Motivational Differences in Problem Video Game Play. Journal of Cyberther- 
apy and Rehabilitation, 2, 139-149.

[5] Griffiths, M.D. (2005) A “Components” Model of Addiction within a Biopsychosocial Framework. Journal of Substance Use, 10, 191-197. http://dx.doi.org/10.1080/14659890500114359

[6] Gentile, D.A., Swing, E.L., Lim, C.G. and Khoo, A. (2012) Video Game Playing, Attention Problems, and Impulsiveness: Evidence of Bidirectional Causality. Psychology of Popular Media Culture, 1, 62-70. http://dx.doi.org/10.1037/a0026969

[7] Haagsma, M.C., Pieterse, M.E. and Peters, O. (2012) The Prevalence of Problematic Video Gamers in the Netherlands. Cyberpsychology, Behavior and Social Networking, 15, 162-168. http://dx.doi.org/10.1089/cyber.2011.0248

[8] Lemmens, J.S., Valkenburg, P.M. and Peter, J. (2009) Development and Validation of a Game Addiction Scale for Adolescents. Media Psychology, 12, 77-95. http://dx.doi.org/10.1080/15213260802669458

[9] Mentzoni, R.A., Brunborg, G.S., Molde, H., Myrseth, H., Skouverøe, K.J.M. and Hetland, J. (2011) Problematic Video Game Use: Estimated Prevalence and Associations with Mental and Physical Health. Cyberpsychology, Behavior and Social Networking, 14, 591-596. http://dx.doi.org/10.1089/cyber.2010.0260

[10] Rehbein, F., Kleimann, M. and Mössle, T. (2010) Prevalence and Risk Factors of Video Game Dependency in Adolescence: Results of a German Nationwide Survey. Cyberpsychology, Behavior and Social Networking, 13, $269-277$. http://dx.doi.org/10.1089/cyber.2009.0227

[11] Mehroof, M. and Griffiths, M.D. (2010) Online Gaming Addiction: The Role of Sensation Seeking, Self-Control, Neuroticism, Aggression, State Anxiety, and Trait Anxiety, Cyberpsychology. Behaviour and Social Networking, 13, 314316. http://dx.doi.org/10.1089/cyber.2009.0229

[12] Peters, C.S. and Malesky, L.A. (2008) Problematic Usage among Highly-Engaged Players of Massively Multiplayer Online Role Playing Games. Cyberpsychology \& Behavior: The Impact of the Internet, Multimedia and Virtual Reality on Behavior and Society, 11, 481-484. http://dx.doi.org/10.1089/cpb.2007.0140

[13] Walther, B., Morgenstern, M. and Hanewinkel, R. (2012) Co-Occurrence of Addictive Behaviours Related to Substance Use, Gambling and Computer Gaming. European Addiction Research, 18, 167-174. http://dx.doi.org/10.1159/000335662

[14] King, D.L., Delfabbro, P.H. and Griffiths, M.D. (2010) Video Game Structural Characteristics: A New Psychological Taxonomy. International Journal of Mental Health and Addiction, 8, 90-106. http://dx.doi.org/10.1007/s11469-009-9206-4

[15] Groves, S.J., Skues, J.L. and Wise, L.Z. (2014) Assessing The Potential Risks Associated with Facebook Game Use. International Journal of Mental Health and Addiction, 12, 670-685. http://dx.doi.org/10.1007/s11469-014-9502-5

[16] Thompson, M. (2013) Facebook Reveals New Growth Stats; 20 Percent of Web Users Play Games Every Day. http://www.insidesocialgames.com/2013/03/26/facebook-reveals-new-growth-stats-20-percent-of-web-users-play-gam es-every-day/

[17] Maiberg, E. (2013) Top 25 Facebook Games of May 2013. http://www.insidesocialgames.com/ 2013/05/01/the top-25-facebook-games-of-may-2013/

[18] Elliott, L., Golub, A., Ream, G. and Dunlap, E. (2012) Video Game Genre as a Predictor of Problem Use. Cyberpsychology, Behavior and Social Networking, 15, 155-216. http://dx.doi.org/10.1089/cyber.2011.0387

[19] King, D.L., Delfabbro, P.H. and Griffiths, M.D. (2010) The Role of Structural Characteristics in Problematic Video Game Play: An Empirical Study. International Journal of Mental Health and Addiction, 9, 320-333. http://dx.doi.org/10.1007/s11469-010-9289-y

[20] Lee, J., Lee, M. and Choi, I.H. (2012) Social Network Games Uncovered: Motivations and Their Attitudinal and Behavioral Outcomes. Cyberpsychology, Behavior and Social Networking, 15, 643-648. http://dx.doi.org/10.1089/cyber.2012.0093

[21] Vorderer, P., Hartmann, T. and Klimmt, C. (2003) Explaining the Enjoyment of Playing Video Games: The Role of Competition. ICEC 2003: Proceedings of the Second International Conference on Entertainment Computing, Pittsburgh, 8-10 May 2003, 1-9.

[22] Horney, K. (1937) The Neurotic Personality of Our Time. Norton, New York.

[23] Ryckman, R.M., Hammer, M., Kaczor, L.M. and Gold, J.A. (1990) Construction of a Hypercompetitive Attitude Scale. Journal of Personality and Assessment, 55, 630-639. http://dx.doi.org/10.1207/s15327752jpa5503\&4 19

[24] Ames, D.R., Rose, P. and Anderson, C.P. (2006) The NPI-16 as a Short Measure For Narcissism. Journal of Research in Personality, 40, 440-450. http://dx.doi.org/10.1016/j.jrp.2005.03.002

[25] Kim, E.J., Namkoong, K., Ku, T. and Kim, S.J. (2008) The Relationship between Online Game Addiction and Aggression, Self-Control and Narcissistic Personality Traits. European Psychiatry: The Journal of the Association of European Psychiatrists, 23, 212-220. http://dx.doi.org/10.1016/j.eurpsy.2007.10.010 
[26] Gray, J.A. (1987) Perspectives on Anxiety and Impulsivity: A Commentary. Journal of Research in Personality, 21, 493-509. http://dx.doi.org/10.1016/0092-6566(87)90036-5

[27] Carver, C.S. and White, T.L. (1994) Behavioral Inhibition, Behavioral Activation, and Affective Responses to Impending Reward and Punishment: The BIS/BAS Scales. Journal of Personality and Social Psychology, 67, 319-333. http://dx.doi.org/10.1037/0022-3514.67.2.319

[28] Carver, C.S. (2004) Negative Affects Deriving from the Behavioural Approach System. Emotion, 4, 3-22. http://dx.doi.org/10.1037/1528-3542.4.1.3

[29] Wood, R.T.A., Griffiths, M.D. and Parke, A. (2007) Experiences of Time Loss among Videogame Players: An Empirical Study. Cyberpsychology and Behaviour, 10, 38-44. http://dx.doi.org/10.1089/cpb.2006.9994

[30] Carleton, R.N., Abrams, M.P. and Asmundson, G.J.G. (2010) The Attentional Resource Allocation Scale (ARAS): Psychometric Properties of a Composite Measure for Dissociation and Absorption. Depression and Anxiety, 27, 775786. http://dx.doi.org/10.1002/da.20656

[31] Karlsen, F. (2011) Entrapment and Near Miss: A Comparative Analysis of Psycho-Structural Elements in Gambling Games and Massively Multiplayer Online Role Playing Games. International Journal of Mental Health and Addiction, 9, 193-207. http://dx.doi.org/10.1007/s11469-010-9275-4

[32] Neuman, L. (2006) Social Research Methods: Qualitative and Quantitative Approaches. 6th Edition, Pearson Education, New York.

[33] King, D.L., Delfabbro, P.H. and Zajac, I.T. (2011) Preliminary Validation of a New Clinical Tool for Identifying Problem Video Game Playing. International Journal of Mental Health Addiction, 9, 72-87. http://dx.doi.org/10.1007/s11469-009-9254-9

[34] Cronbach, L.J. (1951) Coefficient Alpha and the Internal Structure of Tests. Psychometrika, 16, 297-334. http://dx.doi.org/10.1007/BF02310555

[35] King, D.L., Haagsma, M.C., Delfabbro, P.H., Gradisar, M. and Griffiths, M.D. (2013) Toward a Consensus on the Definition of Pathological Video-Gaming: A Systematic Review of Psychometric Assessment Tools. Clinical Psychology Review, 33, 331-342. http://dx.doi.org/10.1016/j.cpr.2013.01.002

[36] Lortie, C.L. and Guitton, M.J. (2013) Internet Addiction Assessment Tools: Dimensional Structure and Methodological Status. Addiction, 108, 1207-1216. http://dx.doi.org/10.1111/add.12202

[37] Luchner, A.F., Houston, J.M., Walker, C. and Houston, A.M. (2011) Exploring the Relationship between Two Forms of Narcissism and Competitiveness. Personality and Individual Differences, 51, 779-782. http://dx.doi.org/10.1016/j.paid.2011.06.033

[38] Ryckman, R.M., Thornton, B. and Butler, J.C. (1994) Personality Correlates of the Hypercompetitive Attitude Scale: Validity Tests of Horney's Theory of Neurosis. Journal of Personality Assessment, 62, 84-94. http://dx.doi.org/10.1207/s15327752jpa6201_8

[39] Raskin, R. and Terry, H. (1988) A Principal-Components Analysis of the Narcissistic Personality Inventory and Further Evidence of its Construct Validity. Journal of Personality and Social Psychology, 54, 890-902. http://dx.doi.org/10.1037/0022-3514.54.5.890

[40] Cooper, A., Gomez, R. and Aucote, H. (2007) The Behavioural Inhibition System and Behavioural Approach System (BIS/BAS) Scales: Measurement and Structural Invariance across Adults and Adolescents. Personality and Individual Differences, 42, 295-305. http://dx.doi.org/10.1016/j.paid.2006.11.023

[41] Demianczyk, A.C., Jenkins, A.L., Henson, J.M. and Conner, B.T. (2014) Psychometric Evaluation and Revision of Carver and White BIS/BAS Scales in a Diverse Sample of Young Adults. Journal of Personality Assessment, 96, 485494. http://dx.doi.org/10.1080/00223891.2013.870570

[42] Marsh, H.W., Wen, Z.L. and Hau, K.-T. (2004) Structural Equation Models of Latent Interactions: Evaluation of Alternative Estimation Strategies and Indicator Variables. Psychological Methods, 9, 275-300. http://dx.doi.org/10.1037/1082-989X.9.3.275

[43] Kline, R. (2011) Principles and Practice of Structural Equation Modelling. 3rd Edition, The Guilford Press, New York.

[44] Dillon, W.R., Kumar, A. and Mulani, N. (1987) Offending Estimates in Covariance Structure Analysis: Comments on the Causes of and Solutions to Heywood Cases. Psychological Bulletin, 101, 126-135.

http://dx.doi.org/10.1037/0033-2909.101.1.126

[45] Chen. K.A., Bollen, A., Paxton, P., Curran, P.J. and Kirby, J.B. (2001) Improper Solutions in Structural Equation Models: Causes, Consequences, and Strategies. Sociological Methods \& Research, 29, 468-508. http://dx.doi.org/10.1177/0049124101029004003

[46] Shaw, A. (2012) Do You Identify as a Gamer? Gender, Race, Sexuality, and Gamer Identity. New Media Society, 14, 28-44. http://dx.doi.org/10.1177/1461444811410394 
[47] Wu, P.C. (2013) Addictive Behaviour in Relation to the Happy Farm Application. Social Behaviour and Personality, 41, 539-554. http://dx.doi.org/10.2224/sbp.2013.41.4.539

[48] Griffiths, M.D. (2010) The Role of Context in Online Gaming Excess and Addiction: Some Case Study Evidence. International Journal of Mental Health Addiction, 8, 119-125. http://dx.doi.org/10.1007/s11469-009-9229-x

[49] Wang, H. and Sun, C.T. (2011) Game Reward Systems: Gaming Experiences and Social Meanings. Proceedings of DiGRA 2011 Conference: Think Design Play, 14-17 September 2011, Hilversum, 1-12. 